\title{
Clasificación de Clavien-Dindo. Herramienta para evaluar las complicaciones tras el tratamiento quirúrgico en niños con apendicitis aguda
}

\author{
O.B. Grijalva Estrada, J.I. Garrido Pérez, F.J. Murcia Pascual, M.R. Ibarra Rodríguez, R.M. Paredes Esteban
}

Unidad de Gestión Clínica de Cirugía Pediátrica. Hospital Universitario Reina Sofía. Córdoba.

\begin{abstract}
RESUMEN
Introducción. En pediatría hay pocos criterios estándares para clasificar y unificar las complicaciones postquirúrgicas y en particular en la apendicectomía, donde, según la literatura, las complicaciones varían de un 5-30\%.

Métodos. Realizamos un estudio retrospectivo, observacional tipo transversal de los pacientes intervenidos por sospecha de apendicitis aguda (AA) durante diciembre de 2018 a enero de 2020. Las complicaciones fueron agrupadas y unificadas según la clasificación de Clavien-Dindo (CD). Se analizan las complicaciones postoperatorias y factores implicados, aplicando estudio estadístico bivariante y multivariante según el programa estadístico SPSS versión 25.

Resultados. Un total de 124 pacientes incluidos. Edad media, 9 años (3-14 años). $62 \%$ niños, $38 \%$ niñas. A todos se les realizó apendicectomía, el 80,6\% apendicectomía abierta, 19,4\% por laparoscopia. El $20 \%$ presentó algún tipo de complicación en el postoperatorio y fueron agrupadas según la clasificación de CD ( $\mathrm{p}=0,002)$. En CD I (64\%) se incluyen la mayoría de las complicaciones. En el grupo CD II (28\%) colecciones tratadas con antibioticoterapia. En el grupo CD IIIb (8\%), colección intraabdominal que requirió reintervención para su drenaje. Los principales factores implicados en la aparición de complicaciones fueron: AA complicadas ( $81 \%$ gangrenosas y perforadas) $(\mathrm{p}<0,001)$, tiempo de evolución $(80 \%>24$ h de evolución) $(\mathrm{p}=0,036)$ que incrementó la estancia media $7 \pm 4$ días $(\mathrm{p}=0,016)$. El valor de proteína $\mathrm{C}$ reactiva $(\mathrm{PCR})(137 \pm 37)$ se relacionó con la identificación de plastrón $(\mathrm{p}<0,001)$, y el valor de PCR $(109 \pm 19)$ con peritonitis $(\mathrm{p}<0,001)$.

Conclusiones. La clasificación de Clavien-Dindo permite utilizar un lenguaje común para clasificar las complicaciones postapendicectomía en cirugía pediátrica, expresando el grado de estas según complejidad del tratamiento utilizado para su resolución.
\end{abstract}

Palabras Clave: Clavien-Dindo; Apendicectomía; Complicaciones.

DOI: $10.54847 /$ cp.2022.01.04

Correspondencia: Dra. Ornella Betzabe Grijalva Estrada.

E-mail: dra.grijalvaornella@gmail.com

Recibido: Mayo 2021

Aceptado: Noviembre 2021
Clavien-Dindo Classification: a TOOL TO ASSESS COMPLICATIONS FOLLOWING SURGICAL TREATMENT IN CHILDREN WITH ACUTE APPENDICITIS

\section{AbSTRaCT}

Introduction. In pediatrics, there are few standard criteria to classify and consolidate postoperative complications, particularly in appendectomy, where according to the literature, complications range from $5 \%$ to $30 \%$.

Methods. A cross-sectional, observational, retrospective study of patients undergoing surgery as a result of suspected acute appendicitis (AA) from December 2018 to January 2020 was carried out. Complications were grouped and consolidated according to the Clavien-Dindo (CD) classification. Postoperative complications and factors involved were analyzed by conducting a bivariate and multivariate statistical study using SPSS statistical software, version 25.

Results. A total of 124 patients were studied. Mean age was 9 years (3-14 years). 62\% were boys, and 38\% were girls. All patients underwent appendectomy $-80.6 \%$ through laparotomy, and $19.4 \%$ through laparoscopy. $20 \%$ of patients had postoperative complications, which were grouped according to the CD classification ( $p=0.002)$. Most complications were included in the CD I group (64\%). Collections treated with antibiotic therapy were included in the CD II group (28\%). Intraabdominal collections requiring re-intervention for drainage purposes were included in the CD IIIb group (8\%). The main factors driving complications were complicated AA (81\% gangrenous and perforated) $(\mathrm{p}<0.001)$ and progression time $(80 \%>24 \mathrm{~h}$ of progression $)(\mathrm{p}=0.036)$, which increased mean hospital stay by $7 \pm 4$ days $(\mathrm{p}=0.016) .137 \pm$ 37 CRP levels were associated with plastron identification $(\mathrm{p}<0.001)$, whereas $109 \pm 19$ CRP levels were associated with peritonitis $(\mathrm{p}<0.001)$.

Conclusions. The Clavien-Dindo classification allows post-appendectomy complications in pediatric surgery to be classified using a common language, by associating complication grade with treatment complexity.

Key Words: Clavien-Dindo; Appendectomy; Complications.

\section{INTRODUCCIÓN}

La apendicitis aguda es una causa habitual de dolor abdominal tanto en adultos como en niños y representa la urgencia quirúrgica más frecuente a nivel mundial(1-4). Los análisis de las relaciones volumen-resultado en la cirugía de adultos han 
encontrado que las características del cirujano y del hospital afectan los resultados del paciente, como la duración de la estancia, los gastos hospitalarios, las complicaciones y la mortalidad. Las investigaciones similares en las especialidades quirúrgicas infantiles son menos numerosas y sus conclusiones son menos claras ${ }^{(5)}$. Varios autores todavía están en un debate sobre una definición universal de "complicaciones" que va desde la definición original de Clavien, "las complicaciones son eventos inesperados no intrínsecos al procedimiento"(6), y la variación de Sokol y Wilson, "una complicación quirúrgica es cualquier resultado indeseable, no intencionado y directo de una operación que afecta al paciente, que no se habría producido si la operación hubiera salido tan bien como se podía esperar"(7). Las complicaciones postoperatorias constituyen un importante indicador de calidad: retardan la evolución de la enfermedad, prolongan la estancia hospitalaria e incrementan los índices de morbilidad y mortalidad ${ }^{(8)}$. A tal efecto, en 1992, Clavien propuso una estratificación de la gravedad de las complicaciones, clasificación posteriormente mejorada por Dindo, dando lugar a la clasificación de Clavien-Dindo en el año 2004(6,9,10).

Esta clasificación está basada en las consecuencias terapéuticas de las complicaciones, que permite un enfoque simple, objetivo y reproducible para la evaluación integral de los resultados quirúrgicos ${ }^{(9,11)}$.

La utilidad de la clasificación de Clavien-Dindo es evaluar la seguridad de los procedimientos, comparar diferentes enfoques basados en una clasificación estandarizada, analizar las curvas de aprendizaje de las técnicas quirúrgicas, usarlo como base del control de calidad interno y para estandarizar errores quirúrgicos, mejorando así la gestión y la prevención ${ }^{(12)}$.

En pediatría hay pocas pautas o criterios estándares para clasificar y unificar las complicaciones postquirúrgicas ${ }^{(5,9,10)}$ y en particular en la apendicectomía ${ }^{(13)}$, donde, según la literatura, las complicaciones varían de un 5-30\% ${ }^{(14)}$. El riesgo de generar una complicación aumenta a medida que aumenta la gravedad del proceso de la enfermedad ${ }^{(15)}$. Estudios previos han identificado factores de riesgo potenciales para generar complicaciones postoperatorias, incluidos la edad, el tiempo hasta la cirugía, la presencia de un apendicolito, factores microbiológicos, ubicación geográfica, raza, nivel socioeconómico e incluso el acceso a seguro médico ${ }^{(16,17)}$. La mayoría de los estudios demuestran mejores resultados quirúrgicos en niños tratados por cirujanos pediátricos en hospitales de especialidades pediátricas ${ }^{(13)}$.

\section{MÉTODOS}

Realizamos un estudio retrospectivo, observacional de tipo transversal de los pacientes intervenidos por diagnóstico clínico o radiológico de apendicitis aguda (AA) desde diciembre de 2018 a enero de 2020, en un hospital pediátrico de tercer nivel.

Todos los pacientes (124 pacientes) fueron tratados siguiendo el modelo terapéutico fast-track en la apendicitis aguda complicada del paciente pediátrico, minimizando así la variabilidad en todas las fases de la atención ${ }^{(18)}$.
Los casos incluidos se identificaron a partir de la historia clínica electrónica del paciente en el programa Diraya del Servicio Andaluz de Salud (DSAS). Los datos demográficos relacionados con la enfermedad y el tratamiento se extrajeron del DSAS, incluida la edad al ingreso, el sexo, procedencia, pruebas de imágenes como ecografía o tomografía, analítica (valor de leucocitos, neutrófilos y valor de la proteína $\mathrm{C}$ reactiva), el tiempo desde el inicio de los síntomas hasta la cirugía (tiempo de evolución), abordaje laparoscópico o laparotomía, estado del apéndice cecal identificada durante la cirugía, diagnóstico anatomopatológico, días de hospitalización y complicaciones postoperatorias. Se excluyeron apendicectomías en el contexto de otra patología digestiva (resección colónica), así como pacientes diagnosticados por técnicas de imagen como plastrón apendicular, ya que en nuestro centro el manejo de estos pacientes es conservador inicialmente, seguido de cirugía diferida $^{(19)}$. Sin embargo, los pacientes que fueron diagnosticados de plastrón apendicular durante la intervención sí se tomaron en cuenta en este estudio. Cabe recalcar que no en todos los pacientes con dolor abdominal en fosa ilíaca derecha se realiza prueba de imagen, siendo muchas veces suficiente para la intervención la alta sospecha diagnóstica de apendicitis aguda. El juicio clínico sigue siendo el factor más importante en el tratamiento de pacientes con sospecha de apendicitis aguda ${ }^{20,28)}$.

Los datos demográficos se resumieron mediante estadística descriptiva (Tabla I). La presencia de complicación se consideró como el resultado primario en el análisis multivariable. Las complicaciones fueron agrupadas y unificadas según la clasificación de Clavien-Dindo (CD) (Tabla II) ${ }^{(21)}$. Se definió como apendicitis complicadas aquellas en las que el apéndice era gangrenoso o perforado a criterio del cirujano, y las peritonitis generalizadas como no complicadas a las formas eritematosa y flemonosa. Se estableció como colección o absceso intraabdominal al acúmulo de líquido intraabdominal postquirúrgico identificado por ecografía o tomografía (Tabla III). Se analizan las complicaciones postoperatorias y factores implicados, aplicando estudio estadístico bivariante y multivariante según el programa estadístico SPSS versión 25.

\section{RESULTADOS}

En el periodo de estudio se intervinieron un total de 124 pacientes por alta sospecha diagnóstica de apendicitis aguda. Edad media 9 años (3-14 años). 62\% niños, 38\% niñas. A todos se les realizó apendicectomía, el 80,6\% por laparotomía, 19,4\% por laparoscopia. Los cirujanos que realizaron las intervenciones son adjuntos y residentes de cirugía pediátrica en un hospital universitario pediátrico de tercer nivel.

Si nos referimos al 20\% de los pacientes que presentó algún tipo de complicación (Tabla III) CD I: 16 pacientes; CD II: 7 pacientes y CD IIIb: 2 pacientes. Del total de pacientes que presentaron alguna complicación, el 52\% (13 pacientes) presentaron vómitos, siendo la complicación más frecuente; dos pacientes requirieron tratamiento antipirético por persistencia 
Tabla I. Datos epidemiológicos.

\begin{tabular}{|c|c|c|c|}
\hline & Todos & Complicados & Análisis estadístico \\
\hline & $\begin{array}{c}\text { Pacientes }(n=124) \\
\text { Media } \pm \text { DS (min-máx. })\end{array}$ & $\begin{array}{c}\text { Pacientes }(n=25) \\
\text { Media } \pm D S(\text { min-máx. })\end{array}$ & $\begin{array}{c}\left.\text { Chi cuadrado } \chi^{2} \text { (valor de } p\right) \\
(p=0,002)\end{array}$ \\
\hline Edad (años) $<5$ años & $\begin{array}{l}9 \pm 2,85 \\
(2-14) \\
n=16\end{array}$ & $\begin{array}{c}8,8 \pm 3,11 \\
(5-13) \\
n=5\end{array}$ & OR: $1,63(1,33-2)^{*}$ \\
\hline Sexo (masculino/femenino) & $77: 47(62 \% / 38 \%)$ & $14: 11(56 \% / 44 \%)$ & \\
\hline 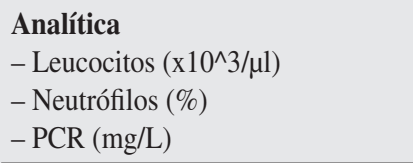 & $\begin{array}{c}15,9 \pm 5 \\
79,6 \pm 8,6 \\
50,3 \pm 68,52\end{array}$ & $\begin{array}{l}17,63 \pm 5,36 \\
82,33 \pm 7,93 \\
70,29 \pm 71,3\end{array}$ & \\
\hline Prueba de imagen preoperatoria & $86(70 \%)$ & $19(76 \%)$ & \\
\hline Procedencia (urbano/rural) & $45 / 79(36 \% / 64 \%)$ & $9 / 16(36 \% / 64 \%)$ & \\
\hline $\begin{array}{l}\text { Horas de evolución } \\
-<12 \mathrm{~h} \\
-12-24 \mathrm{~h} \\
-24-48 \mathrm{~h} \\
-48-72 \mathrm{~h} \\
-72-96 \mathrm{~h}\end{array}$ & $\begin{array}{l}13(10 \%) \\
40(32 \%) \\
37(30 \%) \\
20(17 \%) \\
14(11 \%)\end{array}$ & $\begin{array}{c}0 \\
5(20 \%) \\
10(40 \%) \\
6(24 \%) \\
4(16 \%)\end{array}$ & $\mathrm{p}<0,036^{*}$ \\
\hline Abordaje (LT/LP) & $(100 / 24)(80,6 \% / 19,4 \%)$ & $(20 / 5)(80 \% / 20 \%)$ & \\
\hline $\begin{array}{l}\text { Hallazgos quirúrgicos } \\
\text { - Sin inflamación } \\
\text { - Flemonoso } \\
\text { - Gangrenoso } \\
\text { - Perforado } \\
\text { - Plastrón } \\
\text { - Peritonitis }\end{array}$ & $\begin{array}{l}10(8 \%) \\
60(48 \%) \\
26(20,8 \%) \\
16(12,8 \%) \\
4(3,2 \%) \\
9(7,2 \%)\end{array}$ & $\begin{array}{l}1(4 \%) \\
2(8 \%) \\
8(32 \%) \\
9(36 \%) \\
1(4 \%) \\
4(16 \%)\end{array}$ & $\mathrm{p}<0,001 *$ \\
\hline Estancia media & $3,79 \pm 2,91$ & $7 \pm 4,23$ & $\mathrm{p}<0,016^{*}$ \\
\hline
\end{tabular}

Tabla II. Clasificación de Clavien-Dindo de las complicaciones quirúrgicas.

\begin{tabular}{|c|c|}
\hline Grados & Definiciones \\
\hline I & $\begin{array}{l}\text { Cualquier desviación del curso postoperatorio normal, sin la necesidad de tratamiento farmacológico o intervenciones } \\
\text { quirúrgicas, endoscópicas y radiológicas. Incluye antieméticos, antipiréticos, analgésicos, diuréticos y electrolitos y la } \\
\text { fisioterapia. También incluye las infecciones de la herida abierta }\end{array}$ \\
\hline II & $\begin{array}{l}\text { Requiere tratamiento farmacológico con medicamentos distintos de los autorizados para las complicaciones de grado I. } \\
\text { También se incluyen las transfusiones de sangre y la nutrición parenteral total }\end{array}$ \\
\hline $\begin{array}{l}\text { III } \\
- \text { IIIa } \\
- \text { IIIb }\end{array}$ & $\begin{array}{l}\text { Requiere intervención quirúrgica, endoscópica o radiológico } \\
\text { Intervención que no se da bajo anestesia general } \\
\text { Intervención bajo anestesia general }\end{array}$ \\
\hline $\begin{array}{l}\text { IV } \\
-\mathrm{IVa} \\
-\mathrm{IVb}\end{array}$ & $\begin{array}{l}\text { Complicación potencialmente mortal (incluidas hemorragia cerebral, infarto cerebral, hemorragia subaracnoidea), que } \\
\text { requiere de la gestión de la Unidad de Cuidados Intermedios/Intensivos } \\
\text { Disfunción de un solo órgano (incluyendo la diálisis) } \\
\text { Disfunción multiorgánico }\end{array}$ \\
\hline $\mathbf{V}$ & Muerte de un paciente \\
\hline Sufijo «d» & $\begin{array}{l}\text { Si el paciente padece una complicación en el momento del alta, se añade el sufijo «d» (de discapacidad) al respectivo grado } \\
\text { de complicación. Esta etiqueta indica la necesidad de seguimiento para evaluar la complicación al completo }\end{array}$ \\
\hline
\end{tabular}


Tabla III. Gradación, según clasificación de Clavien-Dindo, descripción de las complicaciones registradas, tratamiento realizado, días de hospitalización y reingreso.

\begin{tabular}{|c|c|c|c|c|c|c|}
\hline $\begin{array}{c}\text { Clasificación } \\
C D\end{array}$ & $\begin{array}{l}\text { Abordaje } \\
\text { quirúrgico }\end{array}$ & $\begin{array}{c}\text { Hallazgo } \\
\text { intraoperatorio }\end{array}$ & Complicación & Tratamiento & $\begin{array}{c}\text { Días de } \\
\text { hospitalización }\end{array}$ & Reingreso \\
\hline I & LT & $P$ & Colección 15 mm & Antibiótico i.v.* & 7 & No \\
\hline I & LT & $\mathrm{Cp}$ & $\begin{array}{c}\text { Colección } 25 \mathrm{~mm} \text { en bolsa escrotal } \\
\text { Orquiepididimitis derecha }\end{array}$ & $\begin{array}{l}\text { Antibiótico v.o. } \\
\text { Analgésicos }\end{array}$ & 5 & No \\
\hline I & LT & $P$ & Diarrea no infecciosa & Sueroterapia & 7 & No \\
\hline I & LT & $\mathrm{NC} \mathrm{f}$ & Colección 22 mm & Antibiótico v.o.* & 2 & No \\
\hline I & LT & $\mathrm{NC} \mathrm{f}$ & Fiebre & Antipiréticos & 4 & No \\
\hline I & LT & $\mathrm{Cg}$ & Vómito & Antieméticos & 6 & No \\
\hline I & LT & $\mathrm{Cp}$ & Vómito & Antieméticos & 5 & No \\
\hline I & LT & $\mathrm{Cp}$ & $\begin{array}{l}\text { Celulitis herida } \\
\text { Hidrocele }\end{array}$ & Antibióticos i.v.* & 10 & No \\
\hline I & LT & $\mathrm{NC} \sin$ & Vómito & Antieméticos & 4 & No \\
\hline I & LP & $\mathrm{Cg}$ & Vómito & Antieméticos & 4 & No \\
\hline I & LT & $\mathrm{Cp}$ & Vómito & Antieméticos & 6 & No \\
\hline I & LT & Plastrón & Fiebre & Antipiréticos & 8 & No \\
\hline I & LT & $\mathrm{Cp}$ & Vómito & Antieméticos & 5 & No \\
\hline I & LP & $\mathrm{Cp}$ & $\begin{array}{l}\text { Dolor } \\
\text { Vómito }\end{array}$ & $\begin{array}{l}\text { Analgésicos } \\
\text { Antieméticos }\end{array}$ & 6 & No \\
\hline I & LT & $\mathrm{Cg}$ & Vómito & Antieméticos & 3 & No \\
\hline I & LT & $\mathrm{Cp}$ & Vómito & Antieméticos & 6 & No \\
\hline II & LT & $\mathrm{Cp}$ & $\begin{array}{c}\text { Colección } 27 \mathrm{~mm} \\
\text { Vómito } \\
\text { Dolor }\end{array}$ & $\begin{array}{l}\text { Antibiótico i.v. } \\
\text { Antieméticos } \\
\text { Analgésicos }\end{array}$ & 8 & No \\
\hline II & LT & $\mathrm{Cg}$ & Neumonía rinovirus & Antivirales & 14 & No \\
\hline II & LP & $\mathrm{Cg}$ & Colección 35 mm & Antibiótico i.v. & 11 & No \\
\hline II & LT & $\mathrm{Cg}$ & $\begin{array}{c}\text { Colección } 30 \mathrm{~mm} \\
\text { Vómito } \\
\text { Dolor }\end{array}$ & $\begin{array}{l}\text { Antibiótico i.v. } \\
\text { Antieméticos } \\
\text { Analgésicos }\end{array}$ & 3 & Sí \\
\hline II & LT & $\mathrm{Cg}$ & Recorte diuresis & $\begin{array}{c}\text { Carga de volumen } \\
\text { NPT }\end{array}$ & 6 & No \\
\hline II & LT & $\mathrm{P}$ & $\begin{array}{l}\text { Colección } 25 \mathrm{~mm} \\
\text { Vómito }\end{array}$ & $\begin{array}{l}\text { Antibiótico i.v. } \\
\text { Antieméticos }\end{array}$ & 15 & No \\
\hline II & LP & $\mathrm{Cg}$ & $\begin{array}{c}\text { Vómito } \\
\text { Colección } 70 \mathrm{~mm}\end{array}$ & $\begin{array}{l}\text { Antieméticos } \\
\text { Antibiótico i.v. }\end{array}$ & 4 & Sí \\
\hline IIIb & LP & $\mathrm{Cp}$ & $\begin{array}{l}\text { Colección } 110 \mathrm{~mm} \\
\text { Derrame pleural derecho }\end{array}$ & $\begin{array}{c}\text { Reintervención } \\
\text { quirúrgica para drenaje }\end{array}$ & 19 & No \\
\hline IIIb & LT & $\mathrm{P}$ & Absceso en pelvis $73 \mathrm{~mm}$ & $\begin{array}{l}\text { Reintervención } \\
\text { quirúrgica para drenaje }\end{array}$ & 13 & No \\
\hline
\end{tabular}

*Mismo tratamiento antibiótico por protocolo de hallazgo intraoperatorio. LT: laparotomía; LP: laparoscopia; NC: no complicada; f: flemonosa; sin: sin inflamación; C: complicado; g: gangrenosa; p: perforada; P: peritonitis; i.v.: intwravenoso.

de fiebre; cuatro pacientes necesitaron rescate analgésico a pesar de tener analgesia pautada; 10 pacientes presentaron colecciones/abscesos intraabdominales que se clasificaron como CD I cuando no se modificó el tratamiento antibiótico según el protocolo postquirúrgico, CD II si se subió al siguiente escalón antibiótico y CD IIIb aquellos que requirieron reintervención quirúrgica bajo anestesia general para su drenaje.

En nuestra serie no presentamos complicaciones descritas en la literatura posterior a apendicectomías como colección en herida quirúrgica u oclusión/suboclusión intestinal. 


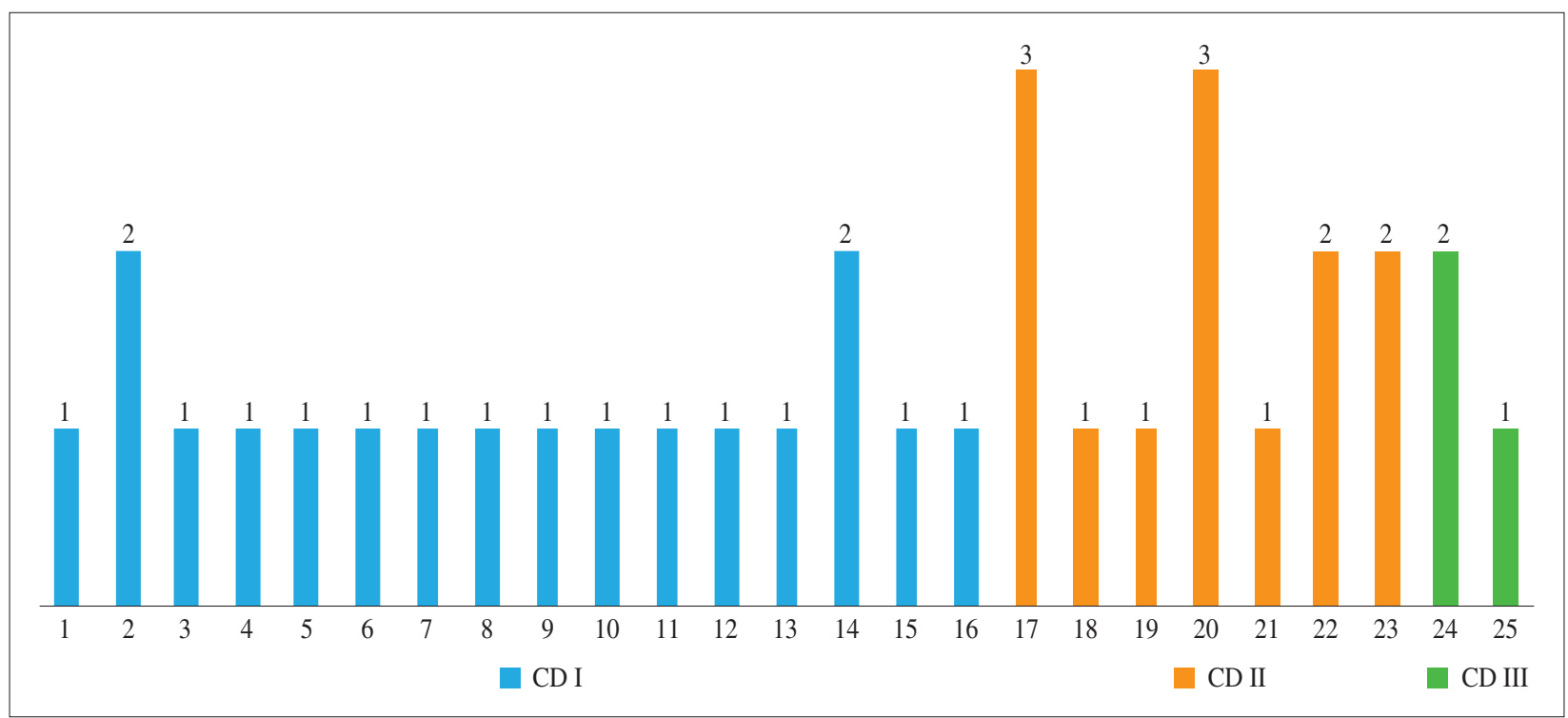

Figura 1. Número de complicaciones por paciente.

La figura 1 muestra el número de complicaciones por paciente. De los pacientes que presentaron algún tipo de complicación, el $80 \%$ refería más de 24 h de evolución del dolor. Los hallazgos quirúrgicos de estos 25 niños fueron apéndices complicadas en 17 pacientes, peritonitis en cuatro pacientes, apéndice no complicado en tres pacientes y un caso de plastrón apendicular, cuyo hallazgo fue intraoperatorio. Los principales factores implicados en la aparición de complicaciones fueron: apendicitis evolucionadas (88\% gangrenosas, perforadas, plastrón y peritonitis) $(\mathrm{p}<0,001)$ y tiempo de evolución $(80 \%>24$ h de evolución) $(\mathrm{p}<0,036)$ que incrementó la estancia media $7 \pm 4$ días $(\mathrm{p}<0,016)$. Un valor de PCR $137 \mathrm{mg} / \mathrm{L} \pm 37 \mathrm{mg} / \mathrm{L}$ se relacionó con la identificación de plastrón ( $\mathrm{p}<0,001)$, y un valor de $109 \mathrm{mg} / \mathrm{L} \pm 19 \mathrm{mg} / \mathrm{L}$ con peritonitis ( $\mathrm{p}<0,001)$ (Fig. 2). En el $20 \%$ el abordaje fue laparoscópico. No hubo relación estadísticamente significativa entre las complicaciones y el abordaje de la intervención (20\% laparoscopias), con la experiencia del cirujano, con los valores de leucocitosis o neutrofilia prequirúrgicos, ni con la procedencia de los pacientes. El $48 \%$ de las piezas quirúrgicas se correlacionaban tanto con el análisis anatomopatológico y los hallazgos en quirófano.

\section{DISCUSIÓN}

Tanto los síntomas y signos clínicos como los hallazgos radiológicos de la apendicitis aguda son extremadamente variables, sobre todo en niños de corta edad (menores de cinco años), en los cuales la inespecificidad de síntomas y signos clínicos, unida a la rápida evolución de dicho proceso y falta de comunicación de los pacientes supone un reto diagnóstico y terapéutico ${ }^{(19)}$.

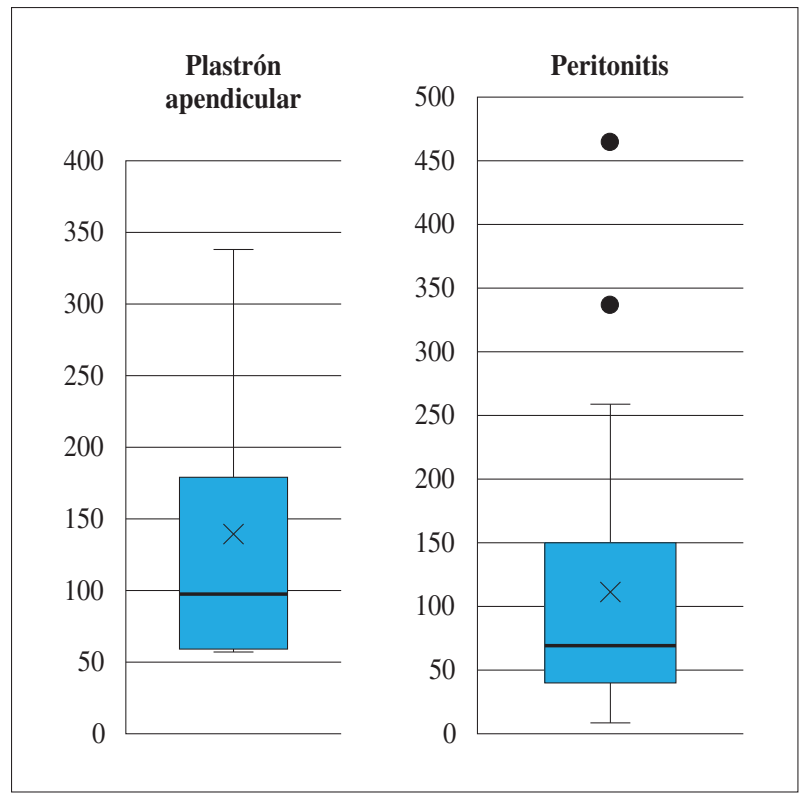

Figura 2. Box Plot con los valores de PCR $(137 \pm 37)$ plastrón apendicular $(\mathrm{p}<0,001)$ y los valores de PCR $(109 \pm 19)$ peritonitis $(\mathrm{p}<0,001)$.

Varios estudios demuestran que los niños pequeños (de 0 a 4 años) presentan apendicitis evolucionadas con mayor frecuencia (OR (odds ratio): 4,9; intervalo de confianza del 95\%: 4,0 a 5,9), en comparación con adolescentes ${ }^{(2,22,23)}$. Se otorga el término apendicitis evolucionadas, tanto por el tiempo de evolución como por el hallazgo intraoperatorio. En nuestra serie se confirmó que a menor edad ( $<5$ años), mayor riesgo de apéndice evolucionada, $80 \%$ en comparación con niños 
mayores o adolescentes (OR: 1,63; intervalo de confianza del 95\%: 1,33 a 2).

Una complicación es un resultado no planeado del procedimiento, algo que causa una desviación de la trayectoria postoperatoria ideal, un evento que induce un cambio en el manejo sometiendo al paciente a intervenciones adicionales. Las complicaciones dependen de la habilidad del cirujano, curva de aprendizaje del procedimiento, comorbilidad del paciente, instalaciones y recursos disponibles. La incidencia de complicaciones postoperatorias sigue siendo el marcador más frecuente de calidad en la cirugía; sin embargo, no hay pautas o criterios estándares para notificar las complicaciones quirúrgicas posteriores a apendicectomías en edad pediátrica.

En nuestro servicio, contamos con un protocolo terapéutico estandarizado en base a los hallazgos en la intervención ${ }^{(18)}$. A pesar de ello, es difícil identificar a los pacientes que presentarán a posteriori algún tipo de complicación. En nuestra serie, la tasa de complicaciones es del $20 \%$, sin registrarse complicaciones de alta gravedad de tipo IV (requieren atención en la Unidad de Cuidados Intermedios/Intensivos) o V (muerte del paciente).

Se han demostrado algunas ventajas con el abordaje laparoscópico, como menor dolor postoperatorio, incisiones más pequeñas, estancias hospitalarias más cortas, un rápido reintegro a las actividades diarias, menor frecuencia de infecciones a nivel de la herida operatoria y menor tasa de adherencias posoperatorias, con la consecuente disminución de complicaciones posoperatorias ${ }^{(24,25)}$. Sin embargo, en nuestra serie, el abordaje quirúrgico no tuvo relación con el desarrollo de complicaciones en el postoperatorio.

En una revisión retrospectiva de 1.255 pacientes intervenidos de apendicitis aguda, determinaron que incluso cuando las muestras de apendicectomía muestran características macroscópicas normales, los análisis histopatológicos pueden proporcionar información clínicamente útil sobre la condición del paciente y ayudar a mejorar el resultado del paciente al revelar una enfermedad previamente no reconocida ${ }^{(26)}$. En otra serie de 69 pacientes encontraron una correlación débil entre el diagnóstico macroscópico del cirujano y los hallazgos anatomopatológicos ${ }^{(27)}$. En nuestra serie, si se toman en cuenta solo los apéndices complicados (gangrenosas y perforadas), el coeficiente kappa mostró solo una correlación débil entre las descripciones de los cirujanos y el estudio anatomopatológico ( $\mathrm{k}=0,28$ IC95\% 0,06-0,50), sin que ello influya en la aparición de complicaciones ( $\mathrm{k}=0,07$ IC95\% -0,05-0,20).

\section{CONCLUSIÓN}

La utilización de la clasificación de Clavien-Dindo podría unificar los criterios de las complicaciones posteriores a la apendicectomía independientemente del abordaje utilizado, y de esta manera basarnos en un sistema estandarizado para notificar complicaciones postquirúrgicas.

\section{BIBLIOGRAFÍA}

1. Solovieva ER, Karaseva OV, Vasilieva MF, Petrichuk SV, Utkina KE. The use of physical factors in the postoperative period with destructive appendicitis in children: the current state of the problem. Vopr Kurortol Fizioter Lech Fiz Kult. 2020; 97(4): 58-65.

2. Singh M, Kadian YS, Rattan KN, Jangra B. Complicated appendicitis: analysis of risk factors in children. Afr J Paediatr Surg. 2014; 11(2): 109-13.

3. Pérez KS, Allen SR. Complicated appendicitis and considerations for interval appendectomy. JAAPA. 2018; 31(9): 35-41.

4. Rentea RM, St Peter SD. Pediatric Appendicitis. Surg Clin North Am. 2017; 97(1): 93-112.

5. McAteer JP, LaRiviere CA, Drugas GT, Abdullah F, Oldham KT, Goldin AB. Influence of surgeon experience, hospital volume, and specialty designation on outcomes in pediatric surgery: a systematic review. JAMA Pediatr. 2013; 167(5): 468-75.

6. Clavien PA, Sanabria JR, Strasberg SM. Proposed classification of complications of surgery with examples of utility in cholecystectomy. Surgery. 1992; 111(5): 518-26.

7. Sokol DK, Wilson J. What is a surgical complication? World J Surg. 2008; 32(6): 942-4.

8. Concha C. Clasificación de las complicaciones postoperatorias. Rev Chil Cir. 2018; 62(3): 314.

9. Dindo D, Demartinez N, Clavien PA. Classification of surgical complications: A new proposal with evaluation in cohort of 6336 patients and results of a survey. Ann Surg 2004; 240: 205-13.

10. Thompson H, Jones C, Pardy C, Kufeji D, Nichols E, Murphy F, et al. Application of the Clavien-Dindo classification to a pediatric surgical network. J Pediatr Surg. 2020; 55(2): 312-5.

11. Espinoza GR, Espinoza GJP. Calidad en cirugía: hacia una mejor comprensión de las complicaciones quirúrgicas [Quality assessment in surgery]. Rev Med Chil. 2016; 144(6): 752-7.

12. Rassweiler JJ, Rassweiler MC, Michel MS. Classification of complications: is the Clavien-Dindo classification the gold standard? Eur Urol. 2012; 62(2): 256-8; discussion 259-60.

13. Tom CM, Friedlander S, Sakai-Bizmark R, Shekherdimian S, Jen $\mathrm{H}$, DeUgarte DA, Lee SL. Outcomes and costs of pediatric appendectomies at rural hospitals. J Pediatr Surg. 2019; 54(1): 103-7.

14. Rolle U, Fahlenbach C, Heidecke CD, Heller G, Meyer HJ, Schuler E, et al. Rates of Complications After Appendectomy in Children and Adolescents: Pediatric Surgical Compared to General Surgical Hospitals. J Surg Res. 2021; 260: 467-74.

15. Abbas PI, Peterson M, Stephens LJ, Rodríguez JR, Lee TC, Brandt ML, et al. Evaluating the effect of time process measures on appendectomy clinical outcomes. J Pediatr Surg. 2016; 51(5): 810-4.

16. Ponsky TA, Huang ZJ, Kittle K, et al. Hospital- and patient-level characteristics and the risk of appendiceal rupture and negative appendectomy in children. JAMA. 2004; 292: 1977-82.

17. Bogle R, McIntyre R, Patel N, Bradnock TJ. Deprivation increases perforation risk in paediatric appendicitis. Arch Dis Child. 2021; 106(4): 392-3.

18. Lasso Betancor CE, Ruiz Hierro C, Vargas Cruz V, Orti Rodríguez RJ, Vázquez Rueda F, et el. Aplicación de un modelo terapéutico fast-track en la apendicitis aguda complicada del paciente pediátrico [Implementation of "fast-track" treatment in paediatric complicated appendicitis]. Cir Pediatr. 2013; 26(2): 63-8. 
19. Murcia Pascual FJ, Garrido Pérez JI, Vargas Cruz V, Betancourth Alvarenga JE, Cárdenas Elías MA, Vázquez Rueda F, et al. Tratamiento quirúrgico o conservador del plastrón apendicular. ¿Influye en la aparición de complicaciones? [Conservative or early surgical management of appendiceal mass. Does it affect the appearance of complications?]. Cir Pediatr. 2015; 28(4): 184-7.

20. Khairy G. Acute appendicitis: is removal of a normal appendix still existing and can we reduce its rate? Saudi J Gastroenterol. 2009; 15(3): 167-70.

21. Merino-Mateo L, Tordable Ojeda C, Cabezalí Barbancho D, Gómez Fraile A. Impact of the COVID-19 pandemic on the surgical activity of Pediatric Urology: analysis of postoperative complications according to the Clavien-Dindo classification. (Table II). Actas Urol Esp (Engl Ed). 2020; 44(10): 659-64.

22. Bratton SL, Haberkern CM, Waldhausen JH. Acute appendicitis risks of complications: age and Medicaid insurance. Pediatrics. 2000; 106(1 Pt 1): 75-8.

23. Ashcraft KW, Holcomb III GW, Murphy JP, editors. Ashcraft's Paediatric Surgery. $5^{\text {th }}$ ed. Philadelphia: Elsevier; 2010. p. 549.
24. Laguzzi MC, Rodríguez F, Costa JM, Chinelli J, Rappa J, Trostchansky J, et al. Abscesos residuales en apendicitis aguda. Comparación entre abordaje laparotómico vs. laparoscópico. Anfamed [Internet]. 2019 [citado 2021 Mayo 24]; 6(1): 170-90.

25. Vahdad MR, Troebs RB, Nissen M, Burkhardt LB, Hardwig S, Cernaianu G. Laparoscopic appendectomy for perforated appendicitis in children has complication rates comparable with those of open appendectomy. J Pediatr Surg. 2013; 48(3): 555-61.

26. Emre A, Akbulut S, Bozdag Z, Yilmaz M, Kanlioz M, Emre R, et al. Routine histopathologic examination of appendectomy specimens: retrospective analysis of 1255 patients. Int Surg. 2013; 98(4): 354-62.

27. Correa J, Jimeno J, Vallverdu H, Bizzoca C, Collado-Roura F, Estalella $\mathrm{L}$, et al. Correlation between intraoperative surgical diagnosis of complicated acute appendicitis and the pathology report: clinical implications. Surg Infect (Larchmt). 2015; 16(1): 41-4.

28. Becker C, Kharbanda A. Acute appendicitis in pediatric patients: an evidence-based review. Pediatr Emerg Med Pract. 2019; 16(9): $1-20$. 